Pacific Journal of Mathematic 


\section{MAPS PRESERVING TRANSLATES OF A FUNCTION}

\section{GLicksberG}

Let $f$ be a bounded continuous function on a topological group $G$ and $\mathscr{O}(f)$ the set of all (left) translates $f_{x}$ of $f$. One might ask what self maps $\phi$ of $G$ have $\mathcal{O}(f) \circ \phi \subset \mathcal{O}(f)$ (so $f_{x} \circ \phi$ is always another translate $f_{y}$ of $f$ ). Since this says $\phi$ maps each translate of a set of constancy $f^{-1}(c)$ into another translate of the set, and indeed a translate independent of $c$, unless $f$ is very special one would expect $\phi$ to be quite rigid, and in fact almost a translation, perhaps on a quotient of $G$.

When $G$ is a compact connected abelian group this is, in essence, the situation if $\phi$ maps $G$ onto itself; alternatively one can take $f \in L^{p}(G), 1 \leqq p<\infty$, and assume $\phi$ is measure preserving and arrive at the same conclusions. In $\S 1$ we determine when $\mathcal{O}(f) \circ \phi \subset \mathcal{O}(f)$ and in $\S 3$ when the distorted orbit $\mathcal{O}(f) \circ \phi$ coincides with another, $\mathcal{O}(g)$, along with some related results. Section 2 is devoted to analogous results on (weakly) almost periodic functions.

Evidently one may as well consider $f$ on the quotient group $G / H$, where $H=\left\{x \in G: f_{x}=f\right\}$, in considering $O(f)$; since $f_{x} \circ \phi=f_{y}$ will hold with $x$ (resp. $y$ ) replaced by any element of the coset $x+$ $H$ (resp. $y+H$ ), one can reduce to the case in which $x \rightarrow f_{x}$ is $1-1$, although producing the corresponding map $\phi$ on $G / H$ is not quite trivial. Consequently we shall begin with the case in which $H$ is trivial and $x \rightarrow f_{x}$ is $1-1$.

THEOREM 1. Suppose $G$ is a compact connected abelian group, $\dot{\phi}$ a map of $G$ onto itself, and $\mathcal{O}(f) \circ \phi \subset \mathcal{O}(f)$ for some $f \in C(G)$ for which $x \rightarrow f_{x}$ is $1-1$ (or alternatively, for some $f \in L^{p}(G)$ while $\phi$ is also measure preserving). Then there is an automorphism $\alpha$ of $G$ and $z_{0}, z_{1} \in G$ for which

$$
\phi(x)=\alpha(x)+z_{0}, \quad x \in G,
$$

while the Fourier series of $f$ is a sum, over the finite orbits of the dual of $\alpha$, of blocks of the form

$$
c \sum_{j=1}^{n}\left(\prod_{k=1}^{j-1} \gamma_{k}\left(z_{1}\right)\right) \gamma_{j}
$$

where $\gamma_{j} \circ \alpha=\gamma_{j+1}, j<n, \gamma_{n} \circ \alpha=\gamma_{1}$, and $\Pi_{1}^{n} \gamma_{j}\left(z_{1}\right)=1$. (Conversely for any such $\alpha, z_{0}, z_{1}$ and $f, \mathcal{O}(f) \circ \phi \subset \mathscr{O}(f)$.) 
In particular when $|\hat{f}|$ has no repeated values, so all the corresponding orbits are singletons, we can conclude $\alpha$ is the identity, so $\phi$ is a translation: for the $1-1$ ness of $x \rightarrow f_{x}$ guarantees $\{\gamma \in \Gamma$ : $\hat{f}(\gamma) \neq 0\}$ separates $G$, and thus generates the dual group $\Gamma$; consequently $\gamma \circ \alpha=\gamma$ for all $\gamma \in \Gamma$.

The result is a simple consequence of the identification [3] of the isometries of compact connected abelian groups, viz.: an isometry $\dot{\phi}$ of a compact connected metric abelian group, under a translation invariant metric, is necessarily of the form (1) for some automorphism $\alpha$. (The hypotheses in [3] include the assumption that $\dot{\phi}$ is onto, but it is well known and easy to prove that an isometry of a compact metric space is automatically onto.)

In order to prove our result we note that the $1-1$ ness of $x \rightarrow f_{x}$ (along with continuity of translation in $C(G)$ or $L^{p}(G)$ ) shows $C(f)$ is a homeomorph of $G$, and thus that $(x, y) \rightarrow\left\|f_{x}-f_{y}\right\|$ is a translation invariant metric defining the topology of $G$. Since $\mathcal{C}(f) \circ \phi \subset \mathscr{\rho}(f)$, for each $x \in G$ we have an element $p(x) \in G$ with $f_{x^{\circ} \circ} \phi=f_{p(x)}$, and since $\phi$ is onto (resp. measure preserving), $p$ is an isometry of the compact metric space $G$ under our translation invariant metric: for

$$
\left\|f_{p(x)}-f_{p(y)}\right\|=\left\|f_{x}^{\circ} \circ \dot{\phi}-f_{y}^{\circ} \circ \dot{\phi}\right\|=\left\|f_{x}-f_{y}\right\| .
$$

Thus we have an automorphism $\alpha$ and $z \in G$ for which $p(x)=\alpha^{-1}(x)+$ $z$, and now $f_{x} \circ \phi(y)=f_{p(x)}(y)$, or $f(\dot{\phi}(y)-x)=f(y-p(x))$, can be written as

$$
f(\dot{\phi}(y)-x)=f\left(y-\alpha^{-1}(x)-z\right),
$$

for all $x$ and $y$ (resp. a.a. $y$ for each $x$ ). An integration (justified by Fubini in our alternate case) yields, on the one hand

$$
\begin{aligned}
\int\langle\gamma, x\rangle f(\dot{\phi}(y)-x) d x & =\langle\gamma, \dot{\phi}(y)\rangle \int \overline{\langle\gamma, \dot{\phi}(y)-x\rangle} f(\dot{\phi}(y)-x) d x \\
& =\langle\gamma, \dot{\phi}(y)\rangle \widehat{f}(\gamma)
\end{aligned}
$$

and on the other

$$
\begin{aligned}
\int\langle\gamma, x\rangle & f\left(y-\alpha^{-1}(x)-z\right) d x=\int\langle\gamma, \alpha(x)\rangle f(y-x-z) d x \\
= & \langle\gamma \circ \alpha, y-z\rangle \int \overline{\langle\gamma \circ \alpha, y-x-z\rangle} f(y-x-z) d x \\
= & \langle\gamma \circ \alpha, y-z\rangle \hat{f}(\gamma \circ \alpha) .
\end{aligned}
$$

Consequently

$$
\hat{f}(\gamma)\langle\gamma, \dot{\phi}(y)\rangle=\hat{f}(\gamma \circ \alpha)\langle\gamma \circ \alpha, y-z\rangle
$$


which shows $\Gamma \backslash \hat{f}^{-1}(0)$ is a union of orbits (under $\gamma \rightarrow \gamma \circ \alpha$ ) each of which is necessarily finite by (3) and Riemann-Lebesgue. Moreover since $\Gamma \backslash \hat{f}^{-1}(0)$ generates $\Gamma$ as we have noted, (3) also implies $\phi$ can be taken continuous in our alternate case. Setting $y=0$ in (3)

$$
\hat{f}(\gamma)\langle\gamma, \phi(0)\rangle=\hat{f}(\gamma \circ \alpha)\langle\gamma,-z\rangle
$$

and so for any $\gamma \notin \hat{f}^{-1}(0)$ we have

$$
\langle\gamma, \phi(y)-\phi(0)\rangle=\langle\gamma \circ \alpha, y\rangle
$$

so $\langle\gamma, \phi(y)\rangle=\langle\gamma, \alpha(y)+\phi(0)\rangle$ for a generating set of $\gamma$, and thus for all $\gamma$ in $\Gamma$. Hence $\phi(y)=\phi(0)+\alpha(y)=\alpha(y)+z_{0}$ as asserted.

Now writing our typical finite orbit as $\gamma_{1}=\gamma_{n} \circ \alpha, \gamma_{2}=\gamma_{1} \circ \alpha, \cdots$, $\gamma_{n}=\gamma_{n-1} \circ \alpha$ and noting that (3) now asserts that $\hat{f}(\gamma)\left\langle\gamma, \alpha(y)+z_{0}\right\rangle=$ $\hat{f}(\gamma \circ \alpha)\langle\gamma, \alpha(y)-\alpha(z)\rangle$, we see that

$$
\hat{f}(\gamma \circ \alpha)=\hat{f}(\gamma)\left\langle\gamma, \alpha(z)+z_{0}\right\rangle
$$

which yields the asserted form for the Fourier series for $f$ when we set $z_{1}=\alpha(z)+z_{0}$.

Corollary 1. If $H=\left\{x \in G: f_{x}=f\right\}$ is nontrivial while the remaining hypotheses of Theorem 1 hold, there is self-map $\bar{\phi}$ of $G / H$ (satisfying $\phi(y)+H \equiv \bar{\phi}(y+H)$ ) which, for the corresponding function $\bar{f}$ on $G / H$, yields $\mathcal{O}(\bar{f}) \circ \bar{\phi}=\mathcal{O}(\bar{f})$, so there is an automorphism $\bar{\alpha}$ of $G / H$ for which

$$
\phi(y)+H=\bar{\phi}(y+H)=\alpha(y+H)+z_{0},
$$

while the Fourier series of $\bar{f}$ (which can be identified with that of $f$ ) has the described form.

In fact this is a corollary of the proof. Our original map $\phi$ suffices to produce our isometry $p$ : as we observed earlier $f_{x} \circ \phi=f_{y}$ implies $f_{x+h^{\circ}} \phi^{\prime}=f_{y+h^{\prime}}, h, h^{\prime} \in H$, so we immediately obtain a self-map $p$ of $G / H$ which satisfies

$$
\left\|\bar{f}_{p(x+H)}-\bar{f}_{p(y+H)}\right\|=\left\|f_{x} \circ \phi-f_{y} \circ \phi\right\|=\left\|f_{x}-f_{y}\right\|=\left\|\bar{f}_{x+H}-\bar{f}_{y+H}\right\| .
$$

Thus we obtain an automorphism $\alpha$ of $G / H$, and a translation, producing $p$ as before; but now from $f_{x} \circ \phi(y)=\bar{f}_{p(x+H)}(y+H)$, we obtain $f(\phi(y)-(x+H))=f\left(y-\alpha^{-1}(x+H)-z\right)$ so that multiplication by $\langle\gamma, x+H\rangle$ for $\gamma \in H^{\perp}$ and integration over $G / H$ now yields

$$
\langle\gamma, \phi(y)+H\rangle=\langle\gamma, \alpha(y+H)+\phi(0)\rangle
$$

for a generating set of $\gamma$ in the dual $H^{\perp}$ of $G / H$. This shows $\bar{\phi}: y+H \rightarrow \phi(y)+H$ is a well defined map on $G / H$, and of course 
the remainder of our argument now applies.

The hypothesis that $\phi$ is onto (for $f \in C(G)$ ) was used to insure that $p$ was an isometry, and not just distance reducing. On the other hand if $\mathscr{O}(f) \circ \phi=O(f)$ then $p$ is distance reducing and onto, and thus necessarily an isometry, by the following ${ }^{1)}$ (no doubt known)

Lemma. Suppose $p$ is a distance reducing map of a compact metric space which is surjective. Then $p$ is an isometry.

We shall use the fact that the iterates of $p$ form an equicontinuous semigroup whose closure $S$ in the topology of uniform convergence is a compact abelian semigroup of distance reducing maps. Trivially every element of $S$ is surjective, while the kernel (least ideal) $K$ of $S$ is a (compact) group [6,4]. Since the identity $e=e^{2}$ of $K$ is surjective it must be the identity map; because $e S \subset$ $K$ each element of $S$, in particular $p$, has a distance reducing inverse, which of course implies $p$ is an isometry.

From the lemma then we have

Corollary 2. For $f \in C(G)$ and $\phi$ a map of $G$ into itself, $\mathscr{O}(f) \circ \phi=\mathscr{O}(f)$ implies the conclusions of Corollary 1.

2. Our results apply to almost periodic and weakly almost periodic functions via a reduction to the above setting, using the almost periodic (Bohr) compactification $G^{a}$ of $G$. For $f \in A P(G)$, the space of almost periodic functions on $G$, let $f^{*}$ denote the extension of $f$ to $G^{a}$.

THEOREM 2. Let $G$ be an abelian group whose Bohr compactification $G^{a}$ is connected, and suppose $\phi$ maps $G$ densely into itself. If $f \in A P(G)$ has $O(f) \circ \phi \subset \mathcal{O}(f)$ (or even in $\mathcal{O}(f)^{-}$, the uniform closure), then there is an automorphism $\alpha$ of $G^{a} / H$, where $H=\{x \in$ $\left.G^{a}: f_{x}^{*}=f^{*}\right\}$, and $z_{0}, z_{1} \in G^{a}$ for which

$$
\phi(y)+H=\alpha(y+H)+z_{0}
$$

while the Fourier series for $f$ is a sum over the finite orbits of the

1) I am indebted to $R$. Burckel for pointing out that a slightly weaker assertion is made by Exercise 13, p. 104, of Kaplansky's Set Theory and Metric Spaces, Allyn and Bacon, 1972, and also for the following proof of the lemma which avoids semigroups: choose $n_{j} \nearrow$ so that the iterates $p^{\left(n_{j}\right)} \rightarrow g$ uniformly; $g$ is of course surjective. For $m_{j}=n_{j+1}-n_{j} \geqq 1$ we have a subsequence $m_{j_{k}}$ for which $p^{\left(m_{j k}\right) \rightarrow h}$ uniformly, and from $p^{\left(m \dot{j}_{\dot{k}}\right)} p^{\left(n_{\left.j_{k}\right)}\right.}=p^{\left({ }^{n} \boldsymbol{j}_{k}+1\right)}$ we conclude $h \circ g=g$ which implies $h$ is the identity map. But trivially $d(x, y)=d(h(x), h(y)) \leqq d(p(x), p(y))$ since $p$ is distance reducing, and we are done. (See the remark added in proof.) 
dual of $\alpha$, of blocks of the form (2).

Our hypothesis implies $\mathscr{O}(f)^{-} \circ \dot{\phi} \subset \mathcal{O}(f)^{-}$, and we can identify $\mathcal{O}(f)^{-}$with $\left\{f_{x}^{*} \mid G: x \in G^{a}\right\}$ (viewing $G$ as a subset of $G^{a}$ of course). Thus there is a self-map $p$ of $G^{a} / H$ with

$$
\left(f_{x+H}^{*} \mid G\right) \circ \dot{\phi}=f_{p(x+H)}^{*} \mid G .
$$

Now the fact that $\phi$ has dense range implies $p$ is an isometry of $G^{a} / H$ under its translation invariant metric

$$
(x+H, y+H) \longrightarrow\left\|f_{x+H}^{*}-f_{y+H}^{*}\right\|
$$

so again we obtain our automorphism determining $p$ and the fact that

$$
f^{*}(\phi(y)-(x+H))=f^{*}\left(y-\alpha^{-1}(x+H)-z\right)
$$

for $y \in G, x \in G^{a}$. So invariant integration over $G^{a} / H$. leads again to the fact that

$$
\phi(y)+H=\alpha(y+H)+\phi(0),
$$

as in the proof of Corollary 1; the asserted form of the Fourier series for $f$ follows as before.

Evidently if $\alpha, H, f$ and $\phi$ satisfy the indicated relation then $O(f) \circ \phi \subset \circlearrowleft(f)^{-}$.

Note that when $G$ is a noncompact abelian group $\Gamma$ is always uncountable while $\Gamma \backslash \bar{f}^{*-1}(0)$ is countable, so $H$, the subgroup of $G^{a}$ orthogonal to $\Gamma \backslash \bar{f}^{*-1}(0)$, is nontrivial. ( $H \cap G$ can easily be trivial, as for $G=R, f(x)=e^{i x}+e^{i x \sqrt{2}}$.)

For $f$ weakly almost periodic $\mathscr{O}(f) \circ \phi \subset \mathcal{O}^{\circ}(f)$ implies $O(f)^{-w} \circ \phi \subset$ $\mathscr{O}(f)^{-w}$, where ${ }^{-w}$ denotes the weak closure; indeed for $g \in \mathcal{C}(f)^{-w}$ we have a net $f_{x} \rightarrow g$ weakly, hence pointwise, so $f_{x} \circ \phi \rightarrow g \circ \dot{\phi}$ pointwise, hence weakly since $g \circ \dot{\phi}$ is the only possible weak cluster point of that net, yielding our assertion. We want to note that this fact has the consequence that

$$
\mathscr{O}\left(f^{a}\right) \circ \phi \subset \mathscr{O}\left(f^{a}\right)^{-} \text {(uniform closure) }
$$

where $f^{a}$ is the almost periodic component of $f$, so that Theorem 3 will apply to relate $f^{a}, \phi$ and an automorphism of a quotient of $G^{a}$ (when $G^{a}$ is connected).

With $W(G)$ the space of weakly almost periodic functions on $G$ we have the decomposition $[1,2]$

$$
W(G)=A P(G) \oplus W_{0}(G)
$$

where $W_{0}(G)$ is the translation invariant space consisting of those 
elements of $W(G)$ having zero in the weak closure of their orbits, while the projections corresponding to (5) are bounded, and thus weakly continuous. Now if $f=f^{a}+f^{0}$, where $f^{0} \in W_{0}(G)$, then we have $x_{\hat{o}}$ in $G$ for which $f_{x_{\tilde{o}}}^{0} \rightarrow 0$ weakly, hence pointwise; moreover we can assume $\left\{f_{x_{\bar{b}}}^{a}\right\}$ converges uniformly to some element $g$ of $O\left(f^{a}\right)^{-}$, so

$$
f_{x_{\tilde{\delta}}} \longrightarrow g+0
$$

pointwise and thus $f_{x_{\hat{o}}} \circ \phi=f_{y_{\hat{o}}} \rightarrow g \circ \phi$ pointwise, so weakly. But since our projection onto $W_{0}(G)$ is weakly continuous and commutes with translation we obtain $f_{y_{\hat{o}}}^{0} \rightarrow 0$ weakly, whence $f_{y_{\hat{\delta}}}^{a} \rightarrow g \circ \phi$ weakly. As the only possible uniform cluster point of the net $\left\{f_{y_{\delta}}^{a}\right\}, g \circ \phi$ is the uniform limit of the net, and we conclude $g \circ \phi \in \mathscr{O}\left(f^{a}\right)^{-}$.

But $f_{x_{\tilde{\delta}}}^{0} \rightarrow 0$ weakly implies $f_{x+x_{\tilde{\delta}}}^{0} \rightarrow 0$ weakly for any $x$, whence by the above argument $f_{x+x_{\hat{o}}}^{a} \rightarrow g_{x}$ and so $g_{x} \circ \phi \in \mathscr{O}\left(f^{a}\right)^{-}$; now the fact that $\mathscr{O}(g)^{-}=\mathscr{O}\left(f^{a}\right)^{-}$for any $g \in \mathscr{O}\left(f^{a}\right)^{-}$(which follows, say, from the fact that $g=f_{x}^{a^{*}} \mid G$ for some $\left.x \in G^{a}\right)$ shows $\mathscr{Q}\left(f^{a}\right)^{-} \circ \phi \subset \mathcal{O}\left(f^{a}\right)^{-}$, as desired.

COROLlary 3. Suppose $G$ is an abelian group with connected Bohr compactification, and $f$ is a weakly almost periodic function with almost periodic component $f^{a}$. If $\phi$ maps $G$ densely into itself while $\mathscr{O}(f) \circ \phi \subset \mathcal{O}(f)^{-w}$ then $\mathcal{O}\left(f^{a}\right) \circ \phi \subset \mathcal{O}\left(f^{a}\right)^{-}$, and the conclusions of Theorem 2 relating $f^{a}$ and $\phi$ apply.

3. We conclude with some variants and remarks. One variant yielding the same conclusion is provided by the hypothesis that $\mathscr{O}(f) \circ \phi \supset \mathcal{O}(f)$, by an almost identical argument. (Without $\phi 1-1$. Others are $\mathscr{O}(f) \circ \phi \subset g+\mathscr{O}(f)$ and the reverse, where $g$ finally turns out to be a constant of mean 0 , so 0 . Moreover Theorem 1 and Corollary 1 hold if only $\mathscr{O}(f) \circ \phi \subset \mathscr{C} \mathscr{O}(f)$, the closed convex hull of the orbit of $f$. Indded this implies $\mathscr{C} \mathscr{O}(f) \circ \phi \subset \mathscr{C} O(f)$, so that composition with $\phi$ provides an isometry of this compact metric space, which is thus necessarily onto; being linear it takes extreme points to extreme points. But each extreme point lies in $\mathscr{O}(f)$, and once one translate is extreme all are by translation, so $\mathscr{O}(f)$ is the

2) In fact, for $F$ the generator of an invariant filter [5], $\left\{f_{x^{\circ}} \phi: x \in F\right\} \subset \mathcal{O}(f)^{-v}$ is an adequate hypothesis. This can be seen most easily from the alternate proof there [5, pp. 420-1], which shows the filter of closed sets on $G^{w}$, the weakly almost periodic compactification of $G$, generated by the sets $(x+F)^{-}$(with $x$ in a dense subgroup $G_{0}$ of $G$ ) contains the least ideal $K=G^{a}$ of $G^{w}$. Since the elements of $W_{0}(G)$ appear on $G^{w}$ as the functions vanishing on $G^{a}$, an argument paralleling the preceding (but using the filter with base $G^{a}$ ) shows we have a $g$ with $g_{x} \in \mathcal{O}\left(f^{a}\right)^{-}$for all $x \in G_{0}$, hence all $x$ in $G$, so our conclusion follows as before. 
set of extreme points, whence $\mathcal{O}(f) \circ \phi \subset \mathcal{O}(f)$ again. The same observation applies to Theorem 2 and Corollary 3, and the argument shows any affine isometry $T$ of $\mathscr{C} O(f)$ is of the form $T f_{x}=f_{\alpha(x)+z}$ where $\alpha$ is an automorphism of $G /\left\{x: f_{x}=f\right\}$ for which $\left\|f_{x}-f\right\| \equiv$ $\left\|f_{\alpha(x)}-f\right\|$, or $^{3)}\left\|f_{x}-f\right\| \equiv\left\|(f \circ \alpha)_{x}-f \circ \alpha\right\|$.

As our next variant we note that we can handle one special case of the more general question of when $\mathscr{O}(f) \circ \phi \subset \mathcal{O}(g)$ can occur. Let $H_{f}$ be the subgroup $\left\{x \in G: f_{x}=f\right\}$ of $G$.

THEOREM 3. Suppose $G$ is a compact connected abelian group, $\phi$ a map of $G$ onto itself, and $\mathcal{O}(f) \circ \phi=\mathcal{O}(g)$ for some $f, g \in C(G)$ (or alternatively, for some $f, g \in L^{p}(G)$ while $\phi$ is also measure preserving). Then there is an isomorphism $\hat{\alpha}$ of the subgroup $H_{f}^{\perp}$ of $\Gamma$ orthogonal to $H_{f}$ into $\Gamma$ for which $H_{g}^{\perp}=\hat{\alpha} H_{f}^{\perp}$, so that the dual map $\alpha: G / H_{g} \rightarrow G / H_{f}$ is an onto isomorphism, while for $\pi: G \rightarrow G / H_{g}$ the natural map,

$$
g=f_{x_{0}} \circ \alpha \pi
$$

and

$$
\phi(x) \in \phi(0)+\alpha \pi(x), \quad x \in G .
$$

Conversely for any such injection $\hat{\alpha}$ of $H_{\vec{f}}^{+}$into $\Gamma$ for which there is a self-map $\phi$ of $G$ satisfying (8), $g$ defined by (7) has $\mathcal{O}(g)=$ $\bigcirc(f) \circ \phi$.

As will be noted the equality of orbits is essential to the proof.4, Indeed we only have to note that it provides two inverse maps $\rho$ : $G / H_{f} \rightarrow G / H_{g}$ and $\sigma: G / H_{g} \rightarrow G / H_{f}$ satisfying, with an obvious abuse of language,

$$
f_{x} \circ \dot{\phi}=g_{\rho(x)}, \quad g_{y}=f_{\sigma(y)} \circ \dot{\phi} .
$$

Now we define our translation invariant metric $d$ on the compact

3) For $L^{2}$ this last condition amounts to $\Sigma|\hat{f}(\gamma)|^{2}|\langle x, \gamma\rangle-1|^{2} \equiv \Sigma\left|\hat{f}(\gamma \circ \alpha)^{-1}\right|^{2} \mid\langle x, \gamma\rangle-$ $\left.1\right|^{2}$, or $\Sigma\left(|\hat{f}(\gamma)|^{2}-\left|\hat{f}\left(\gamma_{\circ} \alpha^{-1}\right)\right|^{2}\right) \operatorname{Re}\langle x, \gamma\rangle \equiv 0$, so that again $|\hat{f}|^{-1}(C)$ is a finite orbit of the dual of $\alpha$ for $C \neq 0$.

4) In fact it is essential to the result. For example with $G=T^{2}, s_{1}, s_{2}, t_{1}, t_{2} \in T$ and $0 \leq \theta<1$, set $\phi\left(t_{1}, e^{2 \pi i \theta}\right)=\left(t_{1} \cdot e^{2 \pi i \theta^{2}}, h\left(e^{2 \pi i \theta}\right)\right)$ where (say) $h$ is any self-homeomorphism of $T$ (so $\phi$ is onto, and also a homeomorphism), and take $f\left(t_{1}, t_{2}\right)=t_{1}$. Then $f_{\left(s_{1}, s_{2}\right)}=$

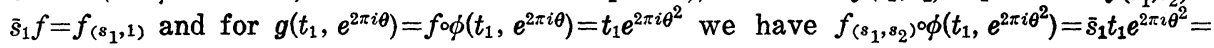
$g_{\left(s_{1} 1\right)}\left(t_{1}, e^{2 \pi i \theta}\right)$, so $\mathscr{O}(f) \circ \phi \subset \mathcal{O}(g)$. On the other hand it is a simple matter to see $H_{g}$ is trivial, so the containment must be proper and no map $\alpha$ as in (8) can exist. ( $H_{f}$ is nontrivial of course; more generally if $H_{f}$ is trivial and $G$ is a finite dimensional torus just the containment suffices since it cannot be proper; for $\rho$ is then a homeomorphism of our torus into another, $G / H_{g}$, of no greater dimension, so invariance of domain for $\boldsymbol{R}^{n}$ guarantees $\rho$ is open, hence onto.) 
connected abelian group $G / H_{f} \times G / H_{g}$ via

$$
d\left((x, y),\left(x^{\prime}, y^{\prime}\right)\right)=\left\|f_{x}-f_{x^{\prime}}\right\|+\left\|g_{y}-g_{y^{\prime}}\right\|
$$

and the self-map $\eta$ of this group by

$$
\eta(x, y)=(\sigma(y), \rho(x)),
$$

and observe $\eta$ is an isometry:

$$
\begin{aligned}
& d\left(\eta(x, y), \eta\left(x^{\prime}, y^{\prime}\right)\right)=d\left((\sigma(y), \rho(x)),\left(\sigma\left(y^{\prime}\right), \sigma\left(x^{\prime}\right)\right)\right) \\
& \quad=\left\|f_{\sigma(y)}-f_{\sigma\left(y^{\prime}\right)}\right\|+\left\|g_{\rho(x)}-g_{\rho\left(x^{\prime}\right)}\right\| \\
& \quad=\left\|f_{\sigma(y)} \circ \phi^{\prime}-f_{\sigma\left(y^{\prime}\right)} \circ \dot{\phi}\right\|+\left\|f_{x^{\circ}} \circ f_{x^{\prime} \circ \boldsymbol{\phi}}\right\| \\
& \quad=\left\|g_{y}-g_{y^{\prime}}\right\|+\left\|f_{x}-f_{x^{\prime}}\right\|=d\left((x, y),\left(x^{\prime}, y^{\prime}\right)\right) .
\end{aligned}
$$

Thus we have an automorphism $\beta$ of $G / H_{f} \times G / H_{g}$ and an element $\left(x_{0}, y_{0}\right)$ of that group for which

$$
\begin{aligned}
(\sigma(y), \rho(x)) & =\beta(x, y)+\left(x_{0}, y_{0}\right) \\
& =\left(\beta_{1}(x, y)+x_{0}, \quad \beta_{2}(x, y)+y_{0}\right) \\
& =\left(\beta_{1}(x, y)+\sigma(0), \quad \beta_{2}(x, y)+\rho(0)\right) .
\end{aligned}
$$

Clearly then $\beta_{1}(x, y)=\alpha(y)$ depends on $y$ alone, $\alpha: G / H_{g} \rightarrow G / H_{f}$ is an isomorphism, and since $\rho$ and $\sigma$ are inverse mappings we obtain $\sigma(y)=\alpha(y)+\sigma(0), \rho(x)=\alpha^{-1}(x)+\rho(0)$, and $\alpha^{-1} \sigma(0)=\rho(0)$. Now (9) says precisely

$$
f(\dot{\phi}(t)-x)=g\left(t-\alpha^{-1}(x)-\rho(0)\right)
$$

(the second equality follows by replacing $x$ by $\alpha[x+\rho(0)]$ ), and repeating our computation of Fourier coefficients we obtain, for $\gamma \in H_{f}=\left(G / H_{f}\right)^{\wedge}$,

$$
\langle\gamma, \phi(t)\rangle \hat{f}(\gamma)=\langle\gamma \circ \alpha, t-\rho(0)\rangle \hat{g}(\gamma \circ \alpha) .
$$

Consequently for $\gamma$ in a generating subset of $H_{f}^{\perp}$, hence for all $\gamma$ in $H_{f}^{\frac{1}{f}}$, we obtain

$$
\langle\gamma, \dot{\phi}(t)-\dot{\phi}(0)\rangle=\langle\gamma \circ \alpha \pi, t\rangle
$$

as before, so $\phi(t) \in \phi(0)+\alpha \pi(t)$ for all $t \in G$ follows. The assertions of Theorem 4 are now easily verified..$^{5}$

The first part of our proof also shows how an isometry $\Phi$ of one orbit $O(f)$ onto another $\mathscr{O}(g)$ can arise. Indeed, using $\Phi$ in place of $f_{x} \rightarrow f_{x} \circ \phi$ we obtain an isomorphism $\alpha$ as above with $\Phi f_{x}=$ $g_{\alpha-1(x)+\rho(0)}$ so that

5) We could equally well consider $\phi: G_{2} \rightarrow G_{1}$ and $\mathscr{O}(f) \circ \dot{\phi}=\mathscr{O}(g)$; the same conclusions follow simply by replacing $G / H_{f}$ and $G / H_{g}$ by $G_{1} / H_{f}$ and $G_{2} / H_{g}$ respectively. 


$$
\begin{aligned}
\left\|f_{x}-f\right\| & =\left\|g_{\alpha^{-1}(x)}-g\right\|=\left\|g_{\alpha^{-1}(x)} \circ \alpha^{-1}-g \circ \alpha^{-1}\right\| \\
& =\left\|\left(g \circ \alpha^{-1}\right)_{x}-g \circ \alpha^{-1}\right\| .
\end{aligned}
$$

Thus $h=g \circ \alpha^{-1}$ has the same displacement function

$$
x \longrightarrow\left\|h_{x}-h\right\|
$$

as $f$. Evidently any $h$ with $\left\|f_{x}-f\right\| \equiv\left\|h_{x}-h\right\|$ (so $H_{h}=H_{f}$ ) and any isomorphism $\alpha^{-1}$ of $G / H_{f}$ onto a quotient of $G$ give rise to $g=$ $h \circ \alpha \pi$ for which $\Phi f_{x}=g_{\alpha^{-1}(x)}$ provides an isometry of orbits, by a permutation of the above norm equality. Consequently we have

Corollary 4. For $G$ compact connected abelian and $f, g$ in $C(G)$ or $L^{p}(G)$, if $\Phi$ is an isometry of $\mathcal{O}(f)$ onto $\mathcal{O}(g)$ then there is an isomorphism $\alpha=G / H_{g} \rightarrow G / H_{f}$ for which $\Phi f_{x}=g_{\alpha^{-1}(x)+y_{0}}$, while $h=g \circ \alpha^{-1} h a s$

$$
\left\|h_{x}-h\right\|=\left\|f_{x}-f\right\|, \quad x \in G .
$$

Conversely for such an $h$ and $\alpha^{-1}$, and $g=h \circ \alpha \pi, \Phi f_{x}=g_{\alpha^{-1}(x)}$ provides such an isometry.

(It should be noted that functions $f$ and $h$ with the same displacement function can have radically unrelated Fourier transforms. For example in $L^{1}[0,2 \pi)$ if $f(x)=\sin x$ and $h$ is any continuous function with say $h=f$ on $[\pi, 2 \pi)$ but whose graph over $(0, \pi)$ is obtained from that of $f$ by horizontal translations varying with height (so $\{x \in[0, \pi): h(x) \geqq y\}$ is an interval of the same length as $\{x \in[0, \pi): f(x) \geqq y\})$ then (12) holds..$\left.^{6}\right)$

A further analogue of our initial result follows when we assume that $\mathcal{O}(f) \circ \phi \subset g \mathcal{O}(f)$, where $g$ is a continuous function of unit modulus (which insures $p$ is again an isometry). Arguing as in Theorem 1 (or Corollary 1) one merely obtains an additional factor $g(y)$ on the right side of (2), viz.,

$$
\hat{f}(\gamma)\langle\gamma, \phi(y)\rangle=\hat{f}(\gamma \circ \alpha \pi)\langle\gamma \circ \alpha \pi, y-z\rangle g(y)
$$

6) It might be worth noting that here the isometry $f_{x \rightarrow} \rightarrow h_{x}$ has no continuous affine extension to the balanced convex set $\mathscr{C O}(f)\left(=-\mathscr{C O O}(f)\right.$ since $\left.f_{\pi}=-f\right)$ : otherwise since $h$ and $f$ are real

$$
\begin{aligned}
\hat{h}(\gamma) \cdot \frac{\gamma+\bar{\gamma}}{2} & =\int h_{x} \operatorname{Re}\langle\gamma, x\rangle d x=\int \Phi f_{x} \operatorname{Re}\langle r, x\rangle d x \\
& =\Phi \int f_{x} \operatorname{Re}\langle\gamma, x\rangle d x=\Phi\left(\hat{f}(\gamma) \frac{\gamma+\bar{\gamma}}{2}\right)
\end{aligned}
$$

so that $\hat{h}$ would be supported by the two characters supporting $\hat{f}$. 
which leads to the fact that

$$
\langle\gamma, \phi(y)-\phi(0)-\alpha \pi(y)\rangle=\frac{g(y)}{g(0)} \text { if } \hat{f}(\gamma) \neq 0 .
$$

(Here again $\alpha$ is an automorphism of $G / H_{f}, \pi: G \rightarrow G / H_{f}$ the natural map.) Now

$$
\beta(y)=\phi(y)-\phi(0)-\alpha \pi(y)
$$

defines a self-map of $G$ whose values on the support $\Gamma \backslash \hat{f}^{-1}(0)$ of $\hat{f}$ are identically $g(y) / g(0)$ for each $y \in G$ by $\left(3^{\prime \prime}\right)$, and so from $\left(3^{\prime}\right)$

$$
\hat{f}(\gamma)\langle\gamma, \phi(0)+\beta(y)+\alpha \pi(y)\rangle=\hat{f}(\gamma \circ \alpha \pi)\langle\gamma, \alpha \pi(y)-\alpha \pi(z)\rangle g(y)
$$

or, since $\langle\gamma, \beta(y)\rangle=g(y) \overline{g(0)}$ if $\hat{f}(\gamma) \neq 0$,

$$
\hat{f}(\gamma \circ \alpha \pi)=\hat{f}(\gamma)\langle\gamma, \phi(0)+\alpha \pi(z)\rangle \overline{g(0)} .
$$

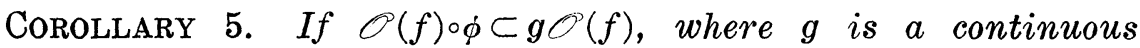
unimodular function, then there is an automorphism $\alpha$ of $G / H_{f}$ and a self-map $\beta$ of $G$ for which

$$
\langle\gamma, \beta(y)\rangle \equiv g(y) \overline{g(0)}
$$

for all $\gamma$ in $\Gamma \backslash \hat{f}^{-1}(0)$ and

$$
\phi(y) \equiv \phi(0)+\alpha(y)+\beta(y)
$$

while the Fourier series of $f$ is a sum, over the finite orbits of the dual of $\alpha$, of blocks, as in (2), now of the form

$$
C \sum_{j=1}^{n} \overline{g(0)^{j}}\left(\prod_{k=1}^{j-1} \gamma_{k}\left(z_{1}\right)\right) \gamma_{k}
$$

Conversely for any such $z$, and $\alpha$, and $\beta$ with values constant on the support of $\hat{f}$, defining $\phi$ from $\alpha, \beta$, and $g$ by $g(y)=g(0)\langle\gamma, \beta(y)\rangle$

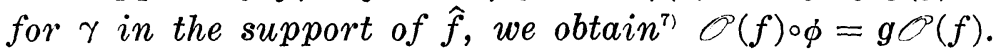

We can also characterize the elements of $\Gamma$.

THEOREM 4. For a compact connected abelian group $G$ and a unimodular function $f, e^{i \theta} f \in \Gamma$ for some $\theta \in \boldsymbol{R}$ iff

$$
\mathscr{O}(f) \cdot \mathscr{O}(f) \subset f \cdot \mathscr{O}(f)
$$

7) $g$ will in fact be constant unless the relations among the elements of $\Gamma \backslash \hat{f}^{-1}(0)$ are special: for $\gamma_{1}, \cdots, \gamma_{k}$ therein if $\Pi \gamma_{i}^{n_{i}}=1$ then $\Sigma n_{i}=0$ since the relation implies $(g(y) / g(0))^{\sum n_{i}}\left\langle\left(\Pi r_{i}^{n_{i}}, \beta y\right)\right\rangle \equiv 1$, and $G$ is connected. For a simple example where $g$ is nonconstant, on $T^{2}$ (written multiplicatively) take $f(s, t)=s+t, \alpha$ the identity say, and

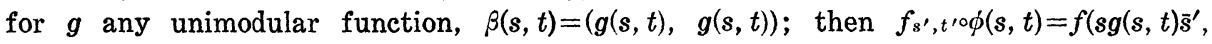
$\left.t g(s, t) \bar{t}^{\prime}\right)=g(s, t)\left(s \bar{s}^{\prime}+t \bar{t}^{\prime}\right)=g(s, t) f_{s^{\prime}, t}(s, t)$. 
or iff

$$
\mathscr{O}(f) \cdot \mathscr{O}(f) \subset f \mathscr{C} \mathscr{O}(f)=\mathscr{C}(f \mathscr{O}(f))
$$

The orbits can be taken in $C(G)$ or $L^{p}(G)$. Trivially a character $f$ satisfies (13) since $f_{x}=\overline{f(x)} f$. On the other hand (13) implies that for $x, y \in G / H_{f}$ we have a unique $p_{y}(x) \in G / H_{f}$ for which $f_{x} \cdot f_{y}=$ $f \cdot f_{p_{y}(x)}$, and clearly $p_{x}(y)=p_{y}(x)$. But since $|f| \equiv 1$,

$$
\begin{aligned}
\left\|f_{p_{y}(x)}-f_{p_{y}\left(x^{\prime}\right)}\right\| & =\left\|f\left(f_{p_{y}(x)}-f_{p_{y}\left(x^{\prime}\right)}\right)\right\|=\left\|f_{x} f_{y}-f_{x^{\prime}} f_{y}\right\| \\
& =\left\|f_{x}-f_{x^{\prime}}\right\|
\end{aligned}
$$

so $p_{y}$ is an isometry of $G / H_{f}$, and again $p_{y}(x)=\alpha_{y}(x)+p_{y}(0)$ for some automorphism $\alpha_{y}$ of $G / H_{f}$. Trivially $f_{0} f_{y}=f \cdot f_{y}$ implies $p_{y}(0)=$ $y$, so

$$
\alpha_{y}(x)+y=p_{y}(x)=p_{x}(y)=\alpha_{x}(y)+x
$$

and setting $x=0$ yields $y=\alpha_{0}(y)$. Now continuity of translation guarantees $y \rightarrow p_{y}$ is continuous into the topology of uniform convergence, so $y \rightarrow \alpha_{y}$ is also; thus for $\gamma \in H_{f}^{+}=\left(G / H_{f}\right)^{\wedge}, y \rightarrow \gamma \circ \alpha_{y}$ is a continuous map of our connected group $G / H_{f}$ into the discrete group $H_{f}^{+}$and so has the constant value $\gamma \circ \alpha_{0}=\gamma$. Consequently $\alpha_{y}$ is always the identity and $p_{y}(x)=x+y$, whence $f_{x} \cdot f_{y}=f \cdot f_{x+y}$. Now

$$
\frac{f(-x)}{f(0)} \frac{f(-y)}{f(0)}=\frac{f(-x-y)}{f(0)}
$$

so $e^{i \theta} f=\overline{f(0)} f \in \Gamma$.

Our proof is completed by the fact that (14) implies (13), by exactly the sort of argument given before Theorem $3 . \quad\left(f_{x} \cdot \mathscr{C}(\mathscr{O}(f)) \subset\right.$ $f \cdot \mathscr{C}(\mathscr{O}(f))$ implies we have an affine isometry of $\mathscr{C}(\mathscr{O}(f))$ into itself, and again it is necessary onto, taking extreme points into extreme points, yielding (13).)

Finally we should note that no result quite as simple as Theorem 1 can hold if $G$ is not connected; for example on $T^{1} \times Z_{3}$ we can take any function $f$ supported by $T^{1} \times\{0\}$ with no vanishing Fourier coefficients, and $\phi$ the map induced by the interchange of the other two cosets. On the other hand in the original setting we can allow our distortion to take place (on Fourier transforms) on $\Gamma$, and then the vital continuity of our invariant metric occurs in a broader context: if $\mu$ is a measure on $G$ with $\hat{\mu} \in C_{0}(\Gamma)$ then any map $\psi$ of $\Gamma$ onto itself for which $\mathscr{O}(\mu)^{\wedge} \circ \mathcal{\psi} \subset \mathcal{O}(\mu)^{\wedge}$ coincides off $\hat{\mu}^{-1}(0)$ with an automorphism $\alpha$ of $H_{\mu}^{\perp} \subset \Gamma$, and $|\hat{\mu}|$ is supported by the finite orbits of $\alpha$ (exactly as in Corollary 1). Indeed we thave $x \rightarrow \hat{\mu}_{x}=x \hat{\mu}$ a continuous map into $C_{0}(\Gamma)$ so that $d(x, y)=\left\|\hat{\mu}_{x}-\hat{\ell}_{y}\right\|_{\infty}$ is a continuous invariant metric on $G / H_{\mu}$ defining its topology. So for the map 
$p$ defined thereon by $\hat{\mu}_{x} \circ \psi=\hat{\mu}_{p(x)}$ we have $z \in G / H_{n}$ and an automorphism $\beta$ of that group for which $p(x)=\beta(x)+z$, and thus

$$
\hat{\mu}_{x}\left(\hat{\gamma}^{\prime}(\gamma)\right)=\langle x, \psi(\gamma)\rangle \hat{\mu}(\dot{\gamma}(\gamma))=\langle\beta(x)+z, \gamma\rangle \hat{\mu}(\gamma) .
$$

(Note that $\hat{\mu}(\gamma) \neq 0$ implies $\gamma \perp H_{: .}$) For the dual $\alpha: H_{\iota^{\perp}} \rightarrow H_{!^{\perp}}^{\perp} \beta$ we therefore have

$$
\langle x, \psi(\gamma)-\alpha(\gamma)\rangle \hat{\mu}(\psi(\gamma))=\langle z, \gamma\rangle \hat{\mu}(\gamma)
$$

for all $x$, and so $\psi^{\prime}=\alpha$ on $\Gamma \backslash \hat{\mu}^{-1}(0) \subset H_{\prime^{\prime}}$ while

$$
\hat{\mu}(\psi(\gamma))=\hat{\mu}(\alpha(\gamma))=\langle z, \gamma\rangle \hat{\mu}(\gamma) ;
$$

consequently $\hat{\mu}$ is supported by the finite orbits of $\alpha$ as earlier while $\hat{\mu} \circ \alpha=\hat{\mu}_{z}$. (As an example where $z \neq 0$, take $\mu(d x)=\sin x d x, \alpha(n)=$ $-n$, and $z=\pi$.) Evidently an analogue of Theorem 3 in particular can be obtained in the same context.

REMARK (added in proof). I am further indebted to $R$. Burckel for locating the Lemma of $\S 1$ in the paper of H. Freudenthal and W. Hurewicz "Dehnungen, verkürzungen, isometrien," Fund. Math., 26 (1936), 120-122.

\section{REFERENCES}

1. R. B. Burckel, Weakly Almost Seriodic Functions on Pemigroups, Gordon and Breach, New York, 1970.

2. K. de Leeuw and I. Glicksberg, Applications of almost periodic compactifications, Acta Math., 105 (1961), 63-97.

3. I. Glicksberg, Some special transformation groups, P.A.M.S., 11 (1960), 315-318.

4. - Convolution semigroups of measures, Pacific J. Math., 9 (1959), 51-67.

5. - Weakly almost periodic functions and generators of invariant filters, Duke Math. J., 42 (1975), 409-422.

6. A. D. Wallace, The structure of topological semigroups, B.A.M.S., 61 (1955), 95-112.

Received December 29, 1978 and in revised form March 29, 1979. Work supported in part by the NSF.

UNIVERSITY OF WASHINGTON

SEATTLE, WA 98195 
.././. ./FrontMatter/paper .pdf 


\section{Pacific Journal of Mathematics \\ Vol. 87, No. $2 \quad$ February, 1980}

Theagenis Abatzoglou, Unique best approximation from a $C^{2}$-manifold in Hilbert space ................................. 233

Gerald Arthur Anderson, $\Lambda$-homology cobordism bundles............. 245

Eric Bedford, Holomorphic mapping of products of annuli in $\mathbf{C}^{n} \ldots \ldots \ldots 271$

Gunnar Carlsson, On the stable splitting of $b o \wedge b o$ and torsion operations in connective $K$-theory .......................... 283

Lester Eli Dubins and David Samuel McIntyre Margolies, Naturally integrable functions ................................. 299

Leo Egghe, The Radon-Nikodým property, $\sigma$-dentability and martingales in locally convex spaces ............................. 313

Irving Leonard Glicksberg, Maps preserving translates of a function ..... 323

Hugh M. Hilden and Robert D. Little, Cobordism of branched covering

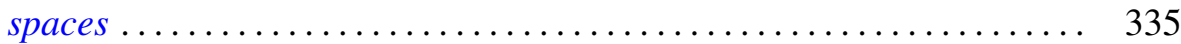

Russell Allan Johnson, Almost-periodic functions with unbounded integral .......................................... 347

Bruce Stephen Lund, The endomorphisms of a Dirichlet algebra ........ 363

John Henry McCleary, Mod $p$ decompositions of H-spaces; another approach........................................ 373

Arlan Bruce Ramsay, Subobjects of virtual groups ................ 389

Thomas R. Savage, Generalized inverses in regular rings ............ 455 Jaak Vilms, On curvature operators of bounded rank ......... 